\title{
ZYGMUND TYPE INEQUALITIES FOR DOUBLE SINGULAR CAUCHY-STIELTJES INTEGRAL
}

\author{
A. GAZIEV
}

Abstract. For the double singular Cauchy-Stiltjes integral over spanning set of a bicylindric domain, a Zygmund type inequality connecting partial and mixed moduli of continuity of the singular integral and its density, is obtained. On this basis, some spaces are constructed, invariant with respect to the double singular integral.

Mathematics subject classification (1991): 30A10.

Key words and phrases: Zygmund inequality, double singular integral, partial nad mixed continuity modulus, invariant spaces.

\section{REFERENCES}

[1] CESARI, L., Sulle serie di Fourier delle funzioni lipschitziane di piu variabili (Italian), Ann. Scuola Norm. Sup. Pisa, II. Ser. 7, 279-295 (1938).

[2] LEKISHVILI, M.M., Conjugate functions of several variables in the class Lip $\alpha$ (Russian), Mat. Zametki 23, 361-372 (1978).

[3] JAK, I.E., On a theorem of L. Cesari. On conjugate functions of two variables (Russian), Dokl. Akad. Nauk. SSSR, 1952, v.87, no 6, 87-880.

[4] Musaev, V.I. and SalaeV, V.V., On conjugate functions of many variables (Russian), Uchen. Zapiski M.V. i SSO Azerb. SSR, ser.fiz-mat. nauk, 1979, no 4, 5-17.

[5] JAK, I.E., On conjugate double trigonometric series (Russian), Matem. sbornik, 1952, v. 31 , no 3.

[6] Dzhvarsheishvili, A.G., On an inequality of A.Zygmund for functions of two variables (Russian), Soobsch. Akad. Nauk Gruz. SSR, 1954, v.15, no 9, 561-568.

[7] KoKILASHVILI, V.M., On some properties of conjugate functions of two variables (Russian), Soobsch. Akad. Nauk. Gruz. SSR, 1965, v.40, no 2.

[8] Magnaragze L.G., On a generalization of Plemeli-Privalov theorem (Russian), Soobsch. Akad. Nauk. Gruz. SSR, 1947, v.4, no 8, 509-516.

[9] Ashurov, R.A. AND SAlaEV V.V., Double singular integral with a continuous density (Russian), Nauch. Trudy. M.V. i SSO Azerb. SSR, ser. fiz.-mat. nauk, 1979, no 6, 29-43.

[10] SalaEV, V.V., Direct and inverse estimates for the Cauchy type singular integral along a closed curve (Russian), Mat. Zametki, 1976, 19, 365-380.

[11] GAZIEV, A.G., The singular operator with the Cauchy-Stiltjes integral along a closed curve (Russian), Izv.AN Uzb SSR,1981, N 1, no 8, 3-9.

[12] GAZIEv, A.G., Study of properties of some linear singular operators, Izv. AN Uzb SSR,1967, no. 2, $7-13$.

[13] GaZIEv, A.G. AND Bubnov, E.A., On Cauchy singular integrals with a continuous density for functions of many variables (Russian), Samarkand State Univ., Samarkand, 1985, Deponierted in UZNIINTI 08.07.85., N $318 \mathrm{Uz}$ - 85 Dep.

[14] KamKe, E., Das Lebesgue-Stieltjes-Integral, Leipzig, Teubner Verlagsgesellschaft VI, 1956, 226 S., 24.

[15] BABA-ZADE, M.A., On a class of continuous functions of two variables (Russian), Uch. Zam. MV i SSO AZSSR, ser. fiz-mat nauk., 1979, no 3, 32-41.

[16] BABAEV, A.A. AND SALAEV, V.V., A one-dimensional singular operator with continuous density along a closed curve (English, Russian original) Sov. Math., Dokl. 14, 598-602 (1973); translation from Dokl. Akad. Nauk SSSR 209, 1257-1260 (1973). 
[17] CheliDze, V.G., On the absolute convergence of double Fourier series (Russian), Dokl. Acad. Nauk. SSSR, v. 54, no 2, 117-119 (1946).

[18] BEZLYUDNEY, A.S., Approximation of periodic functions of two variables by interpolating trigonometric polynomials (Russian), Dokl. Akad. Nauk SSSR, 1949, 65, no 3, 257-260. 